\title{
BMJ Open Quality Improving the documentation quality of point-of-care ultrasound scans in the emergency department
}

\author{
Shadman Aziz (D) , ${ }^{1}$ James Bottomley, ${ }^{1}$ Vasant Mohandas, ${ }^{2}$ Arif Ahmad, ${ }^{2}$ \\ Gemma Morelli, ${ }^{2}$ Sam Thenabadu ${ }^{2}$
}

To cite: Aziz S, Bottomley J, Mohandas V, et al. Improving the documentation quality of point-of-care ultrasound scans in the emergency department. BMJ Open Quality 2020;9:e000636. doi:10.1136/ bmjoq-2019-000636

- Additional material is published online only. To view please visit the journal online (http://dx.doi.org/10.1136/ bmjoq-2019-000636)

Received 24 January 2019 Revised 15 January 2020 Accepted 10 March 2020

\section{Check for updates}

(c) Author(s) (or their employer(s)) 2020. Re-use permitted under CC BY-NC. No commercial re-use. See rights and permissions. Published by BMJ.

${ }^{1}$ School of Medical Education, King's College London, London, UK

${ }^{2}$ Emergency Department, Princess Royal University Hospital, Orpington, UK

Correspondence to Dr Shadman Aziz; shadman.aziz@doctors.net.uk

\section{ABSTRACT}

A point-of-care ultrasound scan (POCUS) is a core element of the Royal College of Emergency Medicine (RCEM) specialty training curriculum. However, POCUS documentation quality can be poor, especially in the time-pressured environment of the emergency department (ED). A survey of 10 junior ED clinicians at the Princess Royal University Hospital (PRUH) found that total POCUS documentation was as low as $38 \%$ in some examinations.

This quality improvement project aimed to increase the coverage and quality of POCUS documentation in the ED. This was done by using a plan-do-studyact (PDSA) regime to improve the quality of POCUS documentation from the original baseline to $80 \%$. There were three discreet PDSA cycles and the interventions included improving education and training about POCUS documentation and the introduction of an original proforma, which incorporated six minimum requirements for POCUS documentation as per the joint RCEM and Royal College of Radiologists (RCR) guidelines for POCUS documentation (patient details, indications, findings, conclusions, signature and date).

The project team audited the quality of all documented scans in the resuscitation department of the PRUH against the RCEM/RCR guidelines at baseline and following three discrete PDSA cycles. This was done over an 8-week period, spanning 696 attendances to the resuscitation area of the ED and 42 documented POCUS examinations.

Quality recording of the six RCEM/RCR elements of POCUS documentation was poor at baseline but improved following three successful PDSA cycles. There was a demonstrated improvement in five of six documentation elements: patient details on POCUS documentation increased from $53.3 \%$ to the $66.7 \%$, indication from $60.0 \%$ to $66.7 \%$, conclusion from $13.0 \%$ to $83.0 \%$, signature from $86.7 \%$ to $100.0 \%$ and date from $46.7 \%$ to $66.7 \%$.

These results suggest that the introduction of a proforma and a vigorous education strategy are effective ways to improve the quality of documentation of ED POCUS.

\section{PROBLEM}

Since 2010, the Royal College of Emergency Medicine (RCEM) has incorporated a point-of-care ultrasound scan (POCUS) as a mandatory element of their higher specialty curriculum. All trainees must be signed off as competent in the four CORE clinical applications (Focused Assessment with Sonography in Trauma (FAST), Assessment of the Abdominal Aorta for Aneurysm, Focused Echocardiography in Life Support, Ultrasound Guided Vascular Access) prior to gaining their certificate of completion of training. ${ }^{1}$

The RCEM Guidance for Level 2 Ultrasound Practice in Emergency Medicine states that every POCUS examination should be formally reported using the format set out by the Royal College of Radiologists (RCR). ${ }^{2}$

- Patient details.

- Indications.

- Findings.

- Conclusion.

- Signed and dated.

The Princess Royal University Hospital (PRUH) is an acute district general hospital in Farnborough, Bromley. The PRUH has a busy emergency department (ED) with over 100000 acute presentations a year. The department has two ultrasound machines used to conduct POCUS.

Documentation of POCUS findings is done freehand into the ED patient note booklet (EDCard), with the exception of trauma, where it is incorporated into a specific trauma booklet. The notes are then scanned into the electronic patient record (EPR) system.

It was noted by the project team that POCUS was often not documented in the patient's notes. Furthermore, the documentation did not meet the standards described previously. This observation led the team to investigate the problem further.

During the planning stages of this quality improvement project (QIP), the project team conducted a survey of POCUS documentation from 10 junior ED clinicians, all either fully accredited in level 1 ultrasound $(n=5)$, or currently completing the experiential $(n=2)$ or competency $(n=3)$ phases of training (online supplementary file 1). Self-reported 
POCUS documentation figures ranged from $38 \%$ to $91 \%$ for the four CORE examinations.

These results suggested a significant amount of scans being performed without documentation, and an unknown number of scans being documented as per the guidance by the RCEM.

The project's SMART aim was to have all documented POCUS in PRUH resus contain $80 \%$ of each of the five key elements of documentation by the end of the 8-week QIP period.

\section{BACKGROUND}

Clinician performed POCUS in the ED is used as an adjunct to other modalities to 'rule-in' life-threatening pathology. The FAST examination has been reported to have sensitivities and specificities for finding free fluid in the abdomen of up to $96 \%$ and $99.7 \%$, respectively, ${ }^{3}$ and an extended FAST examination to look for pneumothorax termed E-FAST has been shown to have sensitivities and specificities of up to $95 \%$ and $99 \%$, respectively. ${ }^{4}$

The low cost of POCUS, its rapid nature and high diagnostic accuracy in detecting potentially life-threatening pathology has led to being incorporated into the curriculum for all ED clinicians completing specialty training in the UK. ${ }^{1}$

However, accurate documentation of POCUS is essential, especially in cases where POCUS has been used to change management, facilitate decision making or been used to compare clinical status across time. Poor documentation affects the quality and safety of patient care as verbal handovers can be misconstrued or the information not given which also has medicolegal implications, and of course, 'If it was not written down, it did not happen'.

Literature examining POCUS documentation is sparse. However, a 2008 study of 42 American ED physicians found that documentation of POCUS was poor, with an average of 1.70 scans being performed per shift, but only $56.4 \%$ of these being documented. Common reasons stated in the paper for poor documentation included 'too many demands on time' and 'requires logging on to a separate programme'. The most popular suggestions among respondents to improve documentation was 'integration into the electronic medical record' and positive recognition/financial reward. ${ }^{5}$

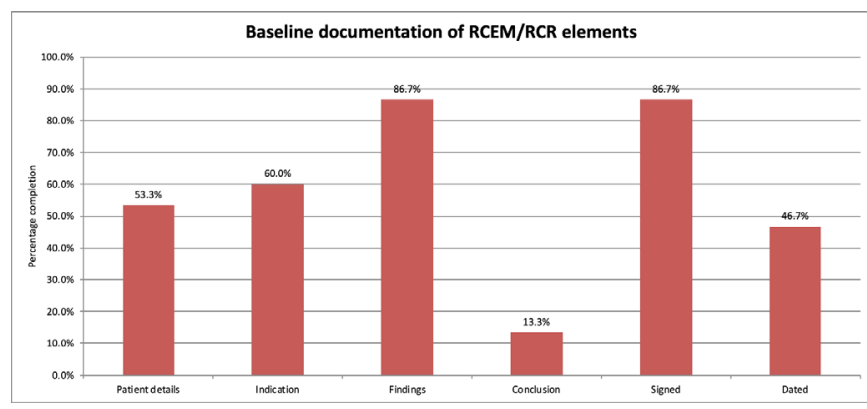

Figure 1 Percentage completion of documentation elements during baseline study.
A thorough literature search yielded limited results in the utility of the use of proformas in POCUS documentation, however, other studies have suggested that proformas can improve documentation of other clinical procedures such as knee arthroscopy. ${ }^{6}$ From the preliminary survey, 8 of 10 of the respondents were amenable to the introduction of a proforma to aid documentation. However, those who objected did not provide reasons for their reluctance to use a proforma (online supplementary file 1 ).

\section{MEASUREMENT}

In order to capture the baseline level of POCUS documentation at the PRUH ED, a baseline study was conducted. Project team members SA and JB completed a retrospective analysis of all the resuscitation department EDCard notes between May 21 and 31, 2018.

Although POCUS is conducted in all areas of the ED (resus, majors, subacute), only patients presenting to resus were included in the study. This was due to time constraints and the high number of patients presenting to ED, making an audit of all patients impractical.

All EDCard notes of resus patients during the timeframe were manually inspected to identify if the patient underwent POCUS. Furthermore, all documented scans were audited against the RCEM/RCR documentation guidelines.

In order to avoid discrepancies and to increase reliability in data recording between the two auditors, strict criteria were agreed on the definitions of each element of the documentation guidelines:

- Patient details: if patient details were recorded anywhere on the EDCard pages where the POCUS was recorded.

- Indication: if a clear clinical question (eg, ? pneumothorax) or valid presentation (abdominal pain) was recorded.

- Findings: if any negative or positive findings were recorded (eg, free fluid found in Morrison's pouch or lack of lung sliding).

- Conclusion: if a clear conclusion was taken from the POCUS documentation (eg, no pneumothorax).

- Signature: if the signature (printed name was acceptable instead of signature) of the completing clinician was recorded anywhere on the EDCard pages where the POCUS was recorded.

- Date: if the date/time of the examination was recorded anywhere on the EDCard pages where the POCUS was recorded.

There were 173 patients who presented to resus during the 10-day baseline study period. Of these 173, 15 POCUS examinations were documented. Of these recordings, documentation of patient details was done in $53 \%$, indication in $60 \%$, findings in $87 \%$, conclusion in $13 \%$, signed in $87 \%$ and dated in $47 \%$ (figure 1).

The high scores for 'findings' and 'signed' acted as realistic targets for improvement and guided the SMART aim target of $80 \%$ for all elements of documentation. 


\section{DESIGN}

The QIP team consisted of two final-year medical students (SA and JB), who designed and led the project, specialty doctor (VM), associate specialist (AA) and ED consultants (GM and ST). VM and SA were involved in the development of the proforma. Data collection and analysis were completed by SA and JB. AA, GM and ST provided supervision for the project. SA and JB drafted the manuscript and critical revision was conducted by GM and ST. Patients were not involved in the planning or design of this project.

A SMART aim was devised to have all documented POCUS in PRUH resus contain $80 \%$ of each of the five key elements of documentation by the end of the 8-week QIP period.

The interventions planned were the introduction of a POCUS proforma, with all the elements of the RCEM/ RCR guidance on documentation of POCUS. This was based on a template proforma included in the RCEM 'Guidance for Level 2 Ultrasound Practice in Emergency Medicine' document. ${ }^{2}$ The project leads consulted various ED staff, who were amenable to the introduction of a proforma, provided it was minimal, user-friendly and non-time consuming due to the busy and stressful nature of ED. The number of proforma uses was recorded as a process measure for this intervention.

The second intervention was the provision of training and regular reminders about the importance of POCUS documentation. The planned interventions included attending ED meetings, teaching sessions and daily handovers; email reminders and the introduction of posters on POCUS documentation in various places in the department. This was done as an attempt to address the issue of buy-in from key stakeholders (junior and senior ED staff involved in ultrasound scanning) and motivation in a very time-pressured and busy department.

As final-year medical students, the project leads (SA and JB) were not permanent members of the ED team at the PRUH, however, the inclusion of substantive consultants increased the sustainability of the project, as they could continue promoting high-quality documentation of POCUS. Furthermore, the implementation of a proforma and posters is a sustainable intervention, as they can be photocopied and continued regardless of changing staff in the department.

\section{STRATEGY}

A series of plan-do-study-act (PDSA) cycles were designed:

\section{PDSA 1}

A POCUS proforma was introduced to the ED teams (online supplementary file 2 ). The proforma included the basic elements of the RCEM/RCR guidelines for POCUS documentation. It was based on the template provided by the RCEM in their 'Guidance for Level 2 Ultrasound Practice in Emergency Medicine' document. ${ }^{2}$
The proforma was designed to be user-friendly, with diagrams for the most common POCUS examinations (FAST/E-FAST, abdominal aorta scan and focused echo) included in a checkbox manner. This was done to minimise the time busy ED clinicians required to fill in the proforma in an attempt to maximise completion. An additional comments section was included for any findings that did not fit the diagrams and for documentation of any other POCUS examinations (eg, transabdominal gynae POCUS and so on). A patient label section was included in the proforma to make it easier for ED clinicians to record the patient details.

Finally, the proformas demonstrated the ultrasound probe positions and placement for the common POCUS examinations. This was included as a training aid for any junior staff conducting POCUS.

The project team attended daily handover meetings and weekly registrar teaching during the baseline study weeks in order to make all clinical ED staff aware of the project and proforma. In addition, two POCUS training sessions were held for the senior Emergency Medicine trainees, which incorporated training on the use of the proformas and a description of the RCEM/RCR guidelines for POCUS documentation. Informal feedback was collected during these encounters to design successive PDSA cycles.

Proformas were attached to the two ED ultrasound machines. During teaching sessions and daily handovers, ED staff were trained on how to fill out the proformas. They were to be filled out for every POCUS examination and inserted into the A\&E EDCard notes for scanning into the EPR system. The project team also made ED admin staff aware of the proformas, and the importance of scanning these into the patient record.

PDSA cycle 1 ran for 10 days between the June 1 and 15 , 2018, with 4 days for the project team to plan and implement the next cycle and complete data analysis. The analysis was identical to the baseline data collection, with auditors SA and JB looking through the resus EDCard notes for the 10-day study period. As the project was looking at the documentation in general, any documentation whether freehand or proforma-based were recorded and audited against the RCEM/RCR guidelines.

\section{PDSA 2}

The study was designed to implement proforma improvements based on clinician feedback during each cycle. It was noted by the project team that some proformas were completed but patient labels were not affixed. This identified a potentially serious patient safety issue whereby scans were documented but not attributable to the patient (although the proforma would be inserted into the EDCard booklet). This was addressed by highlighting the 'affix patient label' box.

Furthermore, the ED staff gave feedback that the writing on the proforma 'findings' was too small. As a result, the diagrams and font size were increased. In addition, the project team highlighted the checkboxes for significant 


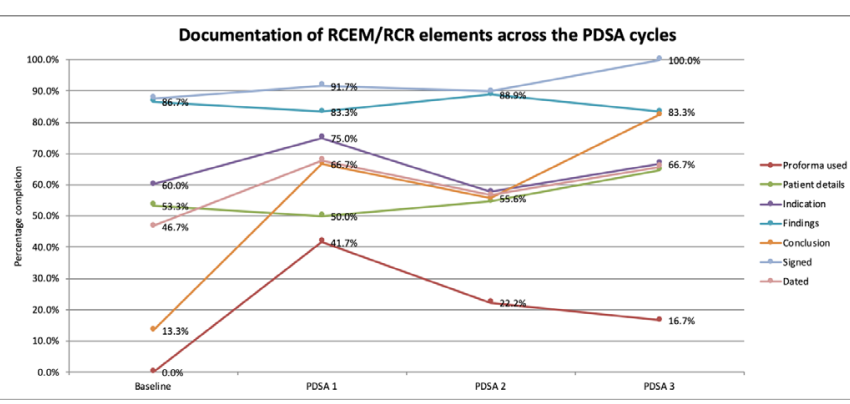

Figure 2 Percentage completion of documentation elements and proforma use from baseline study to PDSA cycle 3. PDSA, plan-do-study-act.

findings in grey (eg, presence of free fluid and absence of lung sliding). This updated version of the proforma replaced all the original proformas on the ultrasound scanners (online supplementary file 2).

The project team continued to attend weekly teaching sessions and daily handovers to make staff aware of the project. This was done to account for those staff who were not present during cycle 1 due to leave, night shifts and to make locum staff aware of the project on a daily basis. There were no new additions to substantive staff during the duration of the QIP.

Finally, email reminders were sent to all ED clinical staff reminding them of the project and the importance of POCUS documentation.

PDSA cycle ran from the June 18 to 28, 2018 with 4 days afterward for the project team to analyse data and plan the next PDSA cycle.

\section{PDSA 3}

The final PDSA cycle ran from the July 2 to 12, 2018.

Staff fed back that they forgot about the proformas and documentation criterion during busy shifts. A poster was designed in order to remind the ED staff to document their scans. It was put up in all clinical areas, staff break rooms and in the admin/office areas (online supplementary file 2).

The project team continued to send reminder emails and attend teaching/handover sessions.

It was also fed back that POCUS proformas were not available at the resus desk, where clinicians would usually complete their paperwork. A tray was set up on the resus desk so that clinicians could find POCUS proformas quickly. The aim of this intervention was to promote the use of the proformas by making them easily accessible to busy clinicians.

\section{RESULTS}

Data were collected from four discrete periods of 10 days each during the baseline study and each of the PDSA cycles. In total, 696 patient encounters were analysed, all in the resus department (baseline: 173, PDSA 1: 173, PDSA 2: 179, PDSA 3: 171).

There was a total of 42 documented POCUS examinations (baseline: 15, PDSA 1: 12, PDSA 2: 9, PDSA 3: 6).
Absolute numbers of documented POCUS examinations decreased during the study.

All documents were included in the analysis both freehand and using the QIP proforma. This was done as the aims of the project were to improve documentation via various methods including reminders at handover and teaching sessions, email reminders and the introduction of the proforma.

All elements of documentation except 'findings' increased throughout the project (figure 2). An itemised breakdown of the results follows:

Patient details: patient details on POCUS documentation increased from $53.3 \%$ to $66.7 \%$ by the end of the project. There were initial concerns during PDSA cycle 1 as some proformas were completed without patient labels affixed, but following the improvement of the proforma, there were no other incidences of this happening.

Indication: during baseline data collection, it was noted that although scans were being documented, the indication for the scan was not present in $60.0 \%$. Although POCUS is a harmless, non-invasive procedure, POCUS can be uncomfortable for patients at times due to some views requiring ultrasound probes to be placed in painful areas or with significant pressure to gain adequate views. Therefore, it is important to describe the indication for the scan. Furthermore, POCUS can take up significant amounts of clinician time and should only be conducted where there is a clear clinical question to be answered. Recording of the indication of scans improved from $60 \%$ to $66.7 \%$ by the end of the project.

Findings: 'findings' was the only element of documentation that did not show improvement and demonstrated a mild decrease by the end of the project from $86.7 \%$ to $83.3 \%$. However, this element was consistently above the SMART target of $80 \%$. However, verbal feedback from ED clinicians stated that the diagrams on the proforma made it easier to record findings and break down the different areas of each POCUS examination.

Conclusion: there was a dramatic increase in the documentation of 'conclusions' from $13.0 \%$ to $83 \%$ at PDSA 3. Documentation of conclusions of POCUS is of significant importance as it provides a clear summary of the scan that can guide further clinical management.

Signed: identification of the clinician(s) performing the scans improved from $86.7 \%$ to $100.0 \%$ by the end of the project. This is important for medicolegal and accountability reasons. A significant positive outcome of the project was that the clinician completing the scan was clearly identifiable in all scans by the end of the QIP.

Dated: finally, the pathology identifiable by POCUS is dynamic and it is important to capture the time that scans were completed. The dates of scans improved significantly from $46.7 \%$ to $66.7 \%$ by the end of the study period.

The SMART aim was not achieved fully as only the 'findings', 'conclusion' and 'signed' elements reached the target $80 \%$.

Separate data analysis was conducted to assess the extent of the introduction of the proforma on the quality 


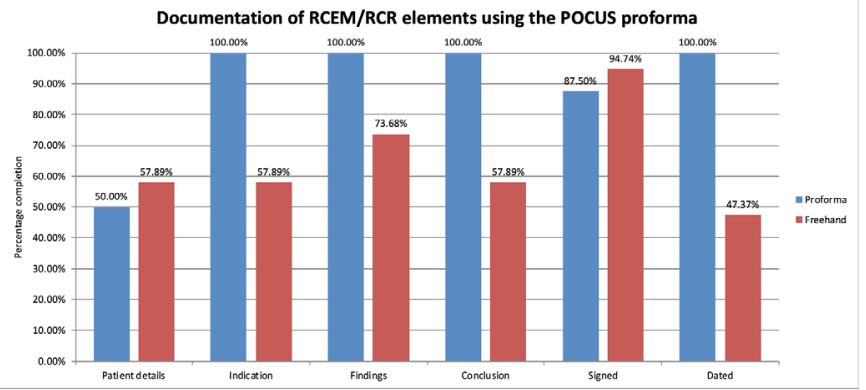

Figure 3 Percentage completion of documentation elements in POCUS proformas compared with freehand documentation. POCUS, point-of-care ultrasound scan; RCEM, Royal College of Emergency Medicine; RCR, Royal College of Emergency Medicine.

of documentation (figure 3). Documentation using proforma performed better than freehand documentation in four of six RCEM/RCR elements, all at compliance of $100.0 \%$. It is likely that the reason for poor performance in the 'patient details' area was due to proformas being completed without patient labels in PDSA cycle 1 .

These results suggest that proforma use can improve the quality of documentation of POCUS in the ED. However, overall proforma completion decreased during the study (figure 2).

\section{LESSONS AND LIMITATIONS}

The major limitation of this QIP was the short time span of the project. The project was designed and led by two final-year medical students (SA and JB) during a 10-week placement at the PRUH. As a result, the project had a significant time constraint. In addition, data were only collected in discrete 10-day periods during each cycle. This was done to allow time for data analysis and implementation of the next PDSA cycles. The result of this was that full continuous data were not collected, subjecting the project to chance as patients requiring POCUS may have presented on the days when data were not being collected.

In addition, this project did not capture the full extent of POCUS at PRUH ED as only patients attending resus were included in the study. This was done for logistical reasons as it would have been impractical for the study team to analyse EDCard notes for every patient attending the ED. However, as resus is a more time-pressured environment than majors/minors and due to the relatively more unwell cohort of patients attending resus, documentation rates may be lower in resus and therefore not generalisable to the rest of the ED.

Furthermore, due to the sparsity of POCUS and the 24/7 nature of A\&E, it was not possible to continuously monitor the use of the ultrasound machine and ensure that all clinicians documented their scans. Therefore, it is likely that POCUS was conducted but not documented at all. This was a problem that the project team identified during the planning stages of the QIP, and one that provided motivation to pursue the project, in an attempt to encourage staff to document all their scans. This was taken into account during the QIP, but it was accepted that it would be impossible to identify all the scans that took place. Therefore, a retrospective approach to analysing documentation was taken. The number of documented scans did decrease throughout the PDSA cycles, however, it is unclear whether this is due to fewer scans being conducted or due to poor documentation.

In addition to this, it was noted that the use of proforma decreased during the study. Although it was demonstrated that proforma completion yielded higher-quality documentation than freehand documentation, the majority of clinicians preferred a freehand approach. One reason for this could have been the availability of proformas, which was addressed in PDSA cycle 3 by adding more proformas to areas where clinicians would sit down and write their notes. Also, freehand documentation could be much quicker than the completion of an additional proforma for busy clinicians in resus. A potential solution to the issues of speed and access for clinicians is to create an electronic proforma. However, this will only be feasible when the department moves to electronic/paperless clerking directly into EPR. Regardless, overall documentation quality improved following the introduction of the proforma, possibly because it acted as a training tool highlighting the important elements of documentation in freehand notes. However, the factors leading to poor uptake of the proforma and its utility as an educational tool were not evaluated.

As the proformas were on a separate sheet of paper, a potential issue was that some completed proformas may not be scanned into EPR, and therefore would not be included in the data collection. However, the project team anticipated this and engaged the ED admin staff in order to minimise the risk of this occurring. There were also concerns that proformas could be scanned into the wrong patient record. For those proformas where the patient label was not affixed, the auditors were able to identify correct upload from the clinical details, name of the sonographer and date/time of the scan. There were no instances of incorrect proforma upload. The risk of this occurring could be mitigated by including the proforma in the patient note booklet rather than a loose sheet of paper or by using an electronic proforma.

Another limitation was that robust evaluation of the documentation education strategy was not carried out. This would have provided valuable process measures to assess the sustainability of the interventions, which this project lacks. On reflection, it would have been useful to survey clinicians attending the teaching sessions and daily handovers, to assess whether they were aware of documentation standards before the study and whether the interventions were likely to change their documentation practice. Furthermore, a repeat survey of the perceived documentation rate was not conducted.

Lastly, this project also highlighted the importance of 'buy-in' from the ED staff. The project team engaged all stakeholders, including junior and senior ED staff. 
A strength of the project is that although the QIP was designed by medical students, the project team included senior clinicians up to consultant level, giving the project legitimacy. However, the ED is a very busy place, with many unwell patients and stressed staff. Staff required significant motivation and regular reminders via email and in-person to complete the additional paperwork of the proforma. A potential solution to this is for the substantive members of the team to deliver documentation training to new staff, thus creating a positive culture of high-quality POCUS documentation from the outset.

Initially, the project team planned to motivate staff to complete the proforma by including a 'carbon copy', which the clinician could keep for their portfolios, however, due to significant cost and the time associated with printing carbon copy forms via third-party companies, this was not pursued. However, given more time and budget, this idea could be leveraged as an incentive to engage with the project.

\section{CONCLUSIONS}

At the time of conducting this QIP, there have been no studies looking into the improvement of documentation of POCUS.

This project did not achieve its SMART aim but has demonstrated that the introduction of a POCUS proforma combined with regular training, reminders and a poster campaign proved to improve over the documentation of POCUS in five of six of the RCEM/RCR elements.

In addition, proforma use was shown to be successful in improving the documentation of POCUS in four of 6 domains described by the RCEM/RCR guidelines. However, proforma use did decline during the study, possibly due to freehand documentation being quicker and the proforma being used as a training tool for documentation.

Significant barriers to improving documentation were identified including time pressure, motivation and buy-in from the clinicians performing the scans.

The project interventions are sustainable, with the proformas and posters established within the ED. Substantive project team members can ensure that there are supplies of proformas available and deliver training to new staff joining the department. Furthermore, there is the possibility of the project being continued by future medical students, as QIPs are integrated into the curriculum at Guy's, King's and St Thomas' School of Medical Education. Finally, as the proforma has now been established at the PRUH, it could be easily replicated at other EDs within the trust and beyond.

Acknowledgements The authors would like to extend their thanks to all junior and senior ED doctors, nurses and admin staff at Princess Royal University Hospital for their involvement and contributions to the project.

Contributors SA and JB: designed and led the project. VM and SA: were involved in the development of the proforma. SA and JB: completed data collection and analysis; drafted the manuscript. AA, GM and ST: provided supervision for the project. GM and ST: conducted critical revision.

Funding The authors have not declared a specific grant for this research from any funding agency in the public, commercial or not-for-profit sectors.

Disclaimer Patients were not involved in the planning or design of this project. Competing interests None declared.

Patient consent for publication Not required.

Provenance and peer review Not commissioned; externally peer reviewed. Data availability statement Data are available upon request.

Open access This is an open access article distributed in accordance with the Creative Commons Attribution Non Commercial (CC BY-NC 4.0) license, which permits others to distribute, remix, adapt, build upon this work non-commercially, and license their derivative works on different terms, provided the original work is properly cited, appropriate credit is given, any changes made indicated, and the use is non-commercial. See: http://creativecommons.org/licenses/by-nc/4.0/.

ORCID iD

Shadman Aziz http://orcid.org/0000-0001-8596-4703

\section{REFERENCES}

1 Royal College of Emergency Medicine. Curriculum for EM core and higher training [Internet]. 2010 Curriculum, 2010. Available: https:// www.rcem.ac.uk/RCEM/Exams_Training/UK_Trainees/Curriculum. aspx?WebsiteKey=b3d6bb2a-abba-44ed-b758-467776a958cd\&hkey= b71ea8aa-ad2f-43fa-b875-0751888ff76c\&2015_Curriculum=3\#2015_ Curriculum

2 Level 2 Working Group. Guidance for level 2 ultrasound practice in emergency medicine, 2008. Available: https://www.rcem.ac.uk/docs/ Training/1.14.6\%20RCEM_Level_2_Guidance-1\%20(1).pdf

3 Savatmongkorngul S, Wongwaisayawan S, Kaewlai R. Focused assessment with sonography for trauma: current perspectives. Open Access Emerg Med 2017;9:57-62.

4 Nandipati KC, Allamaneni S, Kakarla R, et al. Extended focused assessment with sonography for trauma (EFAST) in the diagnosis of pneumothorax: experience at a community based level I trauma center. Injury 2011;42:511-4.

5 Saul T, Siadecki SD, Rose G, et al. 369 a survey evaluation of barriers to provider compliance with point-of-care ultrasound documentation. Ann Emerg Med 2016;68:S141.

6 Mahapatra P, Edmund I. Improving Documentation and Communication Using Operative Note Proformas. BMJ Qual Improv Reports [Internet], 2016. Available: http://qir.bmj.com/lookup/doi/ [Accessed 4 Nov 2018]. 